International Journal of Difference Equations (IJDE).

ISSN 0973-6069, Volume 15, Number 2, (2020) pp. 197-214

(C) Research India Publications

https://dx.doi.org/10.37622/IJDE/15.2.2020.197-214

\title{
Determination of An Optimal Control Strategies for The Discrete Time Addictive Buying Model with The Consumer Confidence Index
}

\author{
Thiti Prasertjitsun $^{1} \quad$ Walailuck Chavanasporn ${ }^{1,2, *}$
}

\author{
Sanoe Koonprasert ${ }^{1,2}$ \\ ${ }^{1}$ King Mongkut's University of Technology North Bangkok, \\ Department of Mathematics, Faculty of Applied Science, \\ Bangkok 10800, Thailand \\ ${ }^{2}$ The Centre of Excellence in Mathematics, CHE, Si Ayutthaya Road, \\ Bangkok 10400, Thailand
}

\begin{abstract}
In this paper, we propose a discrete addictive buying model including two controls: influencer and voucher marketing campaign in order to describe the dynamic of behavior between the classes of buyers, namely, rational buyers, excessive buyers, and addictive buyers. The model also associate with the Consumer Confidence Index (CCI) in Thailand. Identifications of consumer's buying behavior were done by fitting curve techniques based on real databases of CCI, or activity of buyers. We apply Pontryagin's maximum principle in discrete time that is used to characterize the optimal controls and the optimality system is solved by an iterative method. Finally, some numerical simulations are performed to verify the theoretical analysis using MATLAB program.
\end{abstract}

Keywords: Addictive buying, Discrete-time model, Optimal control, Pontryagin's maximum principle..

${ }^{*}$ Corresponding 


\section{INTRODUCTION}

Consumer behavior is an interdisciplinary social science with examining how emotion's attitudes and preferences affect buying behavior. It merge elements from sociology, psychology, social anthropology, economics and marketing, especially behavioral economics. Personal consumer characteristics such as the general life that has a chance to use or support various brands understand the brand and what the brand is trying to understand the needs of a consumers.

The study of consumer behavior also inspect the influences, on the consumer who are involved in buying and using activities or products and included the emotional, psychological and other behavioral responses of consumers.

In the past ten years, consumer behavior models have been developed to confirm various factors affecting consumer behavior and decision making processes [1]. In 2011, I. García and L. Jódar et al. [2] proposed the compartmental model to study the discrete mathematical model of addictive buying using multivariate statistical techniques and sociological approaches for describing consumers buying behavior. In 2016, César M. Silva and Silvério Rosa et al. [4] the authors of this work showed and presented some simulation of the behavior and its discussion with the compartmental model for studying expansion of the regular and referral customers in some corporations.

In 2017, P. Upadhyay and S.K. Upadhyay et al. [5] the authors studied consumer buying behavior via a mathematical model that provides better insights with any product to improve the behavior of buying costumers, the satisfaction of consumer's towards that product. In 2018, Kuldeep Chaudhary et al. [6] the proposed optimal control promotional model policy between a product and the market which first-time and additional repeat purchase buy are assumed to be generated through mass and differentiated promotions. In 2019, Joshua Foster and Cary Deck et al. [7] investigated a combination of theoretical modeling and computational methods, experiments to understand buyers' search behavior in markets.

The Consumer Confidence Index (CCI) determines an indication of developing in the future of saving and consumption, base on the expected financial situation, capability of saving, an unemployment and the general economic situation. An indicator below 100 indicate a pessimistic point of view towards future development in the economy, possibly resulting in a tendency to consume less and save more, but indicator above 100 signals a boost in the consumers confidence towards the future economic situation, as a consequence of which they are spend money on major purchases more inclined and save less. In Thailand, the Consumer Confidence Index (CCI) is presented by the TRADING ECONOMICS [8] and shown in Figure 1.1 


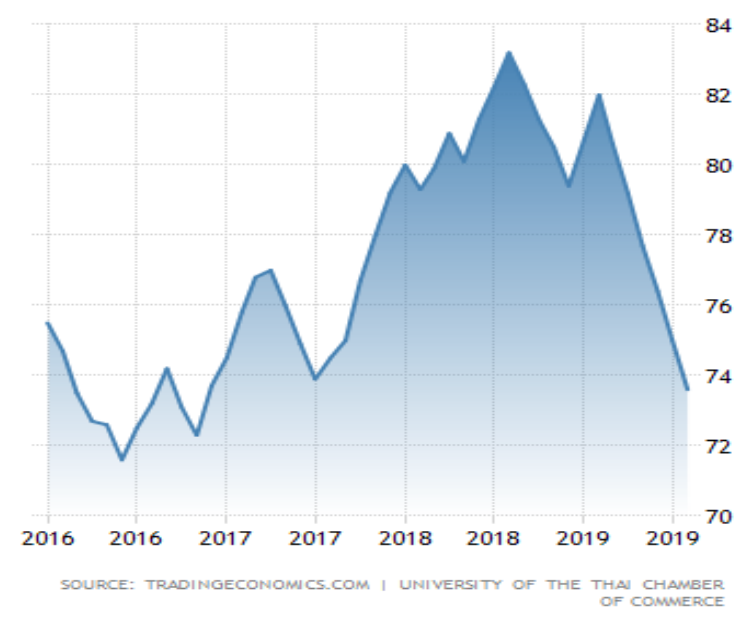

Figure 1.1: Consumer Confidence Index (Thailand).

By fitting curve, the function $\zeta_{i}$ can approximate the consumer confidence index with $R^{2}=0.914752$ as the following function:

$$
\zeta_{i}=\left\{\begin{array}{cc}
-0.00052 i^{6}+0.01514 i^{5}-0.17363 i^{4}+1.01036 i^{3} \\
-3.02377 i^{2}+3.29475 i+74.38999, & 0<i \leq 10, \\
-0.00015 i^{6}+0.01358 i^{5}-0.50430 i^{4}+9.65904 i^{3} & \\
-100.36937 i^{2}+535.63456 i-1072.65173, & 10<i \leq 21, \\
-0.00037 i^{6}+0.05235 i^{5}-3.02822 i^{4}+92.73397 i^{3} & \\
-1585.07127 i^{2}+14334.99059 i-53508.75835, & 21<i \leq 29 \\
0.00069 i^{6}-0.14415 i^{5}+12.55150 i^{4}-580.99331 i^{3} & \\
+15078.06409 i^{2}-208006.40343 i+1191706.76872, & 29<i \leq 39 \\
-0.00004 i^{6}+0.01060 i^{5}-1.20553 i^{4}+72.80569 i^{3} & \\
-2464.48854 i^{2}+44343.77116 i-331300.42338, & i>39 .
\end{array}\right.
$$

The analysis of dynamic systems and the theory of optimal control have been widely used in different fields such as mechanics, engineering, robotics, biology, biomedicine, and medicine. This study is able to predict the behaviour of a model and its control in order to achieve the goals. In 2014, Wandi Ding and Raymond Hendon et al. [9] proposed to applying the theory of discrete-time optimal control for the mathematical modeling of pest control using the extension of Pontryagin's maximum principle. The discrete system and simulations of various cases are provided to show the effectiveness of methods. In 2018, S. Rosa and P. Rebelo et al. [10] applied continuous-time optimal control theory to the problem for a non-autonomous model of ODEs that describes the evolution of the number of customers in some companies for study the best marketing strategy.

In this work, we have been modified a discrete mathematical model [2] and an optimal 
control for the continuous model [3] due to the Consumer Confidence Index (CCI) in Thailand [8] with including the optimal control: influencer and voucher marketing campaign, that describes the dynamics of population and behaviour to an addictive buying. The consumer population in the discrete model can be divided the population denoted by $P$ into three compartments.

Rational buyers $(N)$ who consider the quality, utility, durability, reliability, service, price, etc., of the product and then takes a decision. They purchase only those goods and services which are useful to them and available at reasonable prices.

Excessive buyers $(S)$ who are preoccupied with buying, but who are, at times, able to resist the urge to do so. Excessive buyers are more likely to buy things when they in a good mood state.

Addictive buyers $(A)$ who is buying with a psychological motivation and positive mood state which connected to the construction of an idealized identity, the communication of status or luxury image, and the experience of unlimited power.

We applied the theory of optimal control to achieve these objectives for our proposed model which can determine changing behaviour of addictive buying and the population behaviour regarding buyers. The rest of this paper is organized as follows. In section 2 , we present our discrete mathematical model for the dynamics of the buyer. In section 3 , we present the optimal control problem for the proposed model where we give some results providing the existence of the optimal controls. In section 4, we determine these optimal controls using Pontryagin's maximum principle in discrete time. Numerical simulations are given in section 5. And finally, in section 6, we conclude our paper.

\section{A MATHEMATICAL MODEL}

The discrete diagram describing the dynamics of addictive buying shows the following diagram in Figure 2.1.

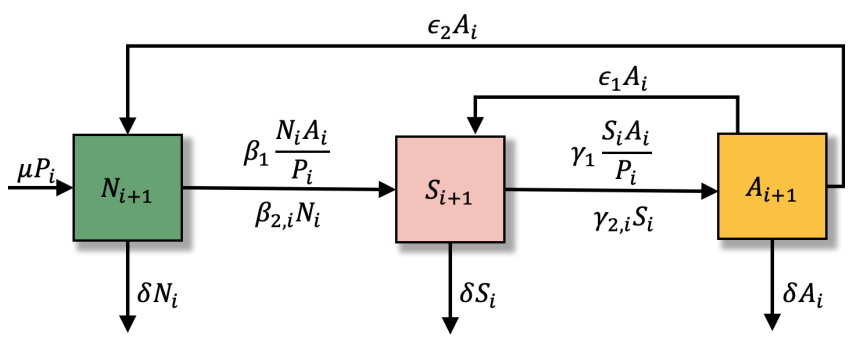

Figure 2.1: Schematic diagram of the three buyer classes in the model at time $i$.

The total population size at time $i$ is denoted by $P_{i}$ with $P_{i}=N_{i}+S_{i}+A_{i}$. The variables $N_{i}$ (Rational buyer), $S_{i}$ (Excessive buyer) and $A_{i}$ (Addictive buyer) are the number of the individuals in the three compartments at time $i$, respectively. The unit of $i$ represents to periods, phases, days, months or years. It depends on the frequency of 
the survey studies as needed. The dynamics of this model are governed by the system of three difference equations:

$$
\begin{aligned}
& N_{i+1}=\left(1-\delta-\beta_{2, i}\right) N_{i}+\mu P_{i}-\beta_{1} \frac{N_{i} A_{i}}{P_{i}}+\epsilon_{2} A_{i} \\
& S_{i+1}=\left(1-\delta-\gamma_{2, i}\right) S_{i}+\beta_{1} \frac{N_{i} A_{i}}{P_{i}}+\beta_{2, i} N_{i}-\gamma_{1} \frac{S_{i} A_{i}}{P_{i}}+\epsilon_{1} A_{i} \\
& A_{i+1}=\left(1-\delta-\epsilon_{1}-\epsilon_{2}\right) A_{i}+\gamma_{1} \frac{S_{i} A_{i}}{P_{i}}+\gamma_{2, i} S_{i}
\end{aligned}
$$

where $N_{0} \geq 0, S_{0} \geq 0$ and $A_{0} \geq 0$ are the given initial states and all parameters are provided by Table 1 .

\begin{tabular}{|c||l||c|}
\hline Parameters & Meaning & Unit \\
\hline$\mu$ & Birth rate & month $^{-1}$ \\
$\delta$ & Death rate & month $^{-1}$ \\
$\beta_{1}$ & Transmission rate due & \\
& to social contact with & month $^{-1}$ \\
& addictive buyers $\left(N_{i} \rightarrow S_{i}\right)$ & \\
$\gamma_{1}$ & Transmission rate due & \\
& to social contact with & month $^{-1}$ \\
$\epsilon_{1}$ & addictive buyer $\left(S_{i} \rightarrow A_{i}\right)$ & \\
& Rate at which reducing & \\
& addictive behavior & \\
& becomes an excessive buyer. & \\
$\epsilon_{2}$ & Rate at which an addictive & \\
& buyer goes to therapy & \\
& change into a rational buyer & \\
\hline
\end{tabular}

Table 2.1: The parameters for Discrete-time model.

Since the parameter $\beta_{2, i}$ represents a rate at which a rational buyer transits to the excessive buyer's subpopulation that related to the consumer confidence index (CCI). We estimate this parameter including $20 \%$ of CCI, $25 \%$ of the population which relates to the behavior of buying, and $2 \%$ of the population which describes emotional instability as the following the parameter

$$
\beta_{2, i}=0.2 * \zeta_{i} *(0.25 * 0.02),
$$

in the Figure 2.2. 


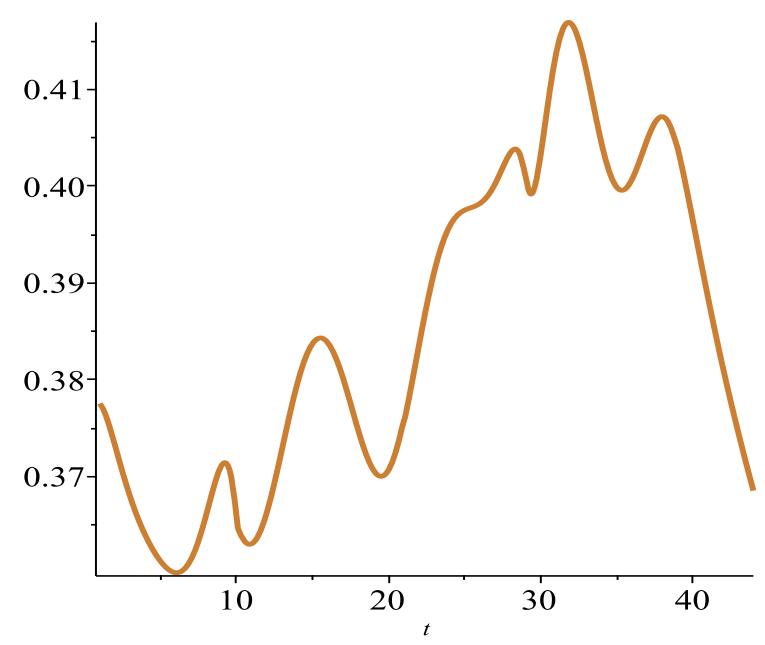

Figure 2.2: Graphs of the parameter $\beta_{2, i}$ relates CCI.

The parameter $\gamma_{2, i}$ represents a rate at which an excessive buyer transits to the addictive buyer's subpopulation that related to the Consumer Confidence Index (CCI) as

$$
\gamma_{2, i}=0.2 * \zeta_{i} *(0.25 * 0.02)+k,
$$

where the function $\zeta_{i}$ represents the consumer confidence index in (1.1) and $k$ is a constant.

\section{THE OPTIMAL CONTROL PROBLEM}

Our objective is this proposed strategy to minimize the campaign controls and control the rational buyer's $\left(N_{i}\right)$, the number of the excessive buyer's $\left(S_{i}\right)$, and the addictive buyer's $\left(A_{i}\right)$. In the model $(2.1)$, we include two controls $u_{1}$ and $u_{2}$. The control $u_{1}$ represents influencer marketing campaign effort to impress the rational buyer's to shop more or becoming the excessive buyer's or the addictive buyer's. The control $u_{2}$ represents the voucher marketing campaign effort ( super sale, big discount, Friday sale ) to motivate the potential rational buyers to buy more goods or products. So, the controlled mathematical system is given by the following system of difference equations as

$$
\begin{aligned}
N_{i+1}= & \left(1-\delta-\beta_{2, i}-u_{2, i}\right) N_{i}+\mu P_{i}-\left(\beta_{1}+u_{1, i}\right) \frac{N_{i} A_{i}}{P_{i}}+\epsilon_{2} A_{i}, \\
S_{i+1}= & \left(1-\delta-\gamma_{2, i}\right) S_{i}+\left(\beta_{1}+\left(1-\alpha_{1}\right) u_{1, i}\right) \frac{N_{i} A_{i}}{P_{i}}+\left(\beta_{2, i}+\left(1-\alpha_{2}\right) u_{2, i}\right) N_{i} \\
& -\gamma_{1} \frac{S_{i} A_{i}}{P_{i}}+\epsilon_{1} A_{i}, \\
A_{i+1}= & \left(1-\delta-\epsilon_{1}-\epsilon_{2}\right) A_{i}+\gamma_{1} \frac{S_{i} A_{i}}{P_{i}}+\gamma_{2, i} S_{i}+\alpha_{1} u_{1, i} \frac{N_{i} A_{i}}{P_{i}}+\alpha_{2} u_{2, i} N_{i},
\end{aligned}
$$


where $N_{0} \geq 0, S_{0} \geq 0, A_{0} \geq 0$ and $\alpha_{1}$ represents the percentage of rational buyers among new customers who have social contact among people who are addictive buying and $\alpha_{2}$ represents the percentage of rational buyers in new customer groups related to the consumer confidence index.

The optimal control problem to minimize the objective functional is given by

$$
\begin{aligned}
\mathcal{J}\left(u_{1}, u_{2}\right)= & \kappa_{1} A_{T}+\kappa_{2} S_{T}+\kappa_{3} N_{T} \\
& +\sum_{i=0}^{T-1}\left(\kappa_{1} A_{i}+\kappa_{2} S_{i}+\kappa_{3} N_{i}+\frac{\kappa_{4}}{2} u_{1, i}^{2}+\frac{\kappa_{5}}{2} u_{2, i}^{2}\right),
\end{aligned}
$$

where the parameters $\kappa_{1}>0, \kappa_{2}>0, \kappa_{3}>0, \kappa_{4}>0$ and $\kappa_{5}>0$ are selected to weigh the relative importance of the cost of cost program, encouragement program, and follow up and psychological support, the cost coefficients respectively.

$$
\mathcal{J}\left(u_{1}^{*}, u_{2}^{*}\right)=\min _{\left(u_{1}, u_{2}\right) \in \mathbf{U}} \mathcal{J}\left(u_{1}, u_{2}\right),
$$

where $\mathbf{U}$ is the set of admissible controls defined by

$$
\begin{array}{r}
\mathbf{U}=\left\{u_{j}=\left(u_{j, 0}, u_{j, 1}, u_{j, 2}, \ldots, u_{j, T-1}\right) \mid 0 \leq u_{j \min } \leq u_{j, i} \leq u_{j \max } \leq 1\right. \\
\text { for } j=1,2, i=0,1,2, \ldots, T-1\} .
\end{array}
$$

The sufficient condition for the existence of an optimal control $\left(u_{1}^{*}, u_{2}^{*}\right)$ for problems Eqs. (3.1) and (3.2) comes from the following theorem.

Theorem 3.1. There exist the optimal controls $u_{1}^{*}, u_{2}^{*}$ such that

$$
\mathcal{J}\left(u_{1}^{*}, u_{2}^{*}\right)=\min _{\left(u_{1}, u_{2}\right) \in \mathbf{U}} \mathcal{J}\left(u_{1}, u_{2}\right)
$$

subject to the control system (3.1) with initial conditions.

Proof. Since the coefficients of the state equations are bounded, so there is a finite number of time steps $(T)$ such that each of three finite sequences

$$
\begin{aligned}
N & =\left(N_{0}, N_{1}, N_{2}, \ldots, N_{T}\right), \\
S & =\left(S_{0}, S_{1}, S_{2}, \ldots, S_{T}\right), \\
A & =\left(A_{0}, A_{1}, A_{2}, \ldots, A_{T}\right),
\end{aligned}
$$

are uniformly bounded for all $\left(u_{1}, u_{2}\right)$ in the control set $\mathbf{U}$, thus the functional $\mathcal{J}\left(u_{1}, u_{2}\right)$ is also bounded for all $\left(u_{1}, u_{2}\right) \in \mathbf{U}$. While $\mathcal{J}\left(u_{1}, u_{2}\right)$ is bounded, the value of $\inf _{\left(u_{1}, u_{2}\right) \in \mathbf{U}} \mathcal{J}\left(u_{1}, u_{2}\right)$ is finite. It applies that there exists a sequence $\left(u_{1}^{j}, u_{2}^{j}\right) \in \mathbf{U}$ such that

$$
\lim _{j \rightarrow \infty} \mathcal{J}\left(u_{1}^{j}, u_{2}^{j}\right)=\inf _{\left(u_{1}, u_{2}\right) \in \mathbf{U}} \mathcal{J}\left(u_{1}, u_{2}\right)
$$


and corresponding the sequence of state $N^{j}, S^{j}, A^{j}$ where

$$
\begin{aligned}
N^{j} & =\left(N_{0}^{j}, N_{1}^{j}, N_{2}^{j}, \ldots, N_{T}^{j}\right), \\
S^{j} & =\left(S_{0}^{j}, S_{1}^{j}, S_{2}^{j}, \ldots, S_{T}^{j}\right), \\
A^{j} & =\left(A_{0}^{j}, A_{1}^{j}, A_{2}^{j}, \ldots, A_{T}^{j}\right) .
\end{aligned}
$$

Since there is a finite number of uniformly bounded sequences such that

$$
\begin{aligned}
\lim _{j \rightarrow \infty}\left(u_{1}^{j}, u_{2}^{j}\right) & =\left(u_{1}^{*}, u_{2}^{*}\right) \\
\lim _{j \rightarrow \infty}\left(N_{0}^{j}, N_{1}^{j}, \ldots, N_{T}^{j}\right) & =\left(N_{0}^{*}, N_{1}^{*}, \ldots, N_{T}^{*}\right)=N^{*} \\
\lim _{j \rightarrow \infty}\left(S_{0}^{j}, S_{1}^{j}, \ldots, S_{T}^{j}\right) & =\left(S_{0}^{*}, S_{1}^{*}, \ldots, S_{T}^{*}\right)=S^{*} \\
\lim _{j \rightarrow \infty}\left(A_{0}^{j}, A_{1}^{j}, \ldots, A_{T}^{j}\right) & =\left(A_{0}^{*}, A_{1}^{*}, \ldots, A_{T}^{*}\right)=A^{*}
\end{aligned}
$$

so there exist $\left(u_{1}^{*}, u_{2}^{*}\right) \in \mathbf{U}$ and $N^{*}, S^{*}, A^{*} \in \mathbf{R}^{T+1}$. Finally, the finite dimensional structure of the system (3.1) and the objective function $\mathcal{J}\left(u_{1}, u_{2}\right)$, so $\left(u_{1}^{*}, u_{2}^{*}\right)$ is an optimal control with corresponding states $N^{*}, S^{*}$, and $A^{*}$. Therefore $\inf _{\left(u_{1}, u_{2}\right) \in \mathbf{U}} \mathcal{J}\left(u_{1}, u_{2}\right)$ is achieved.

\section{FORMULATION OF THE OPTIMAL CONTROLS}

We apply the Pontryagin's maximum principle for discrete version [9, 11-14] which the concept introduces the adjoint function to attach the system of difference equations to the objective functional resulting in the formation the Hamiltonian function. By Hamiltonian principle, the problem of seeking the control to optimize the objective functional subject to the state difference equation with initial condition can find the control to optimize Hamiltonian pointwise.

Now we have the Hamiltonian $\mathcal{H}_{i}$ at time step $i$, defined by

$$
\begin{aligned}
\mathcal{H}_{i}= & \kappa_{1} A_{i}+\kappa_{2} S_{i}+\kappa_{3} N_{i}+\frac{\kappa_{4}}{2} u_{1, i}^{2}+\frac{\kappa_{5}}{2} u_{2, i}^{2} \\
& +\lambda_{1, i+1} N_{j, i+1}+\lambda_{2, i+1} S_{j, i+1}+\lambda_{3, i+1} A_{j, i+1}
\end{aligned}
$$

where $j=1,2,3$ in the system of difference equations (3.1) of the $j^{\text {th }}$ state variable at time step $i+1$.

Theorem 4.1. Given an optimal control $\left(u_{1}^{*}, u_{2}^{*}\right) \in \mathbf{U}$ and solutions $N_{i}^{*}, S_{i}^{*}$ and $A_{i}^{*}$ of 
corresponding state system (3.1), there exist functions, $\lambda_{1}, \lambda_{2}$ and $\lambda_{3}$, satisfying

$$
\begin{aligned}
\lambda_{1, i}= & \kappa_{3}+\lambda_{1, i+1}\left[1+\mu-\delta-\beta_{2, i}-u_{2, i}-\left(\beta_{1}+u_{1, i}\right) \frac{A_{i}\left(S_{i}+A_{i}\right)}{P_{i}^{2}}\right] \\
& +\lambda_{2, i+1}\left[\beta_{2, i}+\left(1-\alpha_{2}\right) u_{2, i}+\left(\left(\beta_{1}-\left(\alpha_{1}-1\right) u_{1, i}\right)\left(S_{i}+A_{i}\right)+\gamma_{1} S_{i}\right) \frac{A_{i}}{P_{i}^{2}}\right] \\
& +\lambda_{3, i+1}\left[\left(\alpha_{1} u_{1, i}\left(S_{i}+A_{i}\right)-\gamma_{1} S_{i}\right) \frac{A_{i}}{P_{i}^{2}}+\alpha_{2} u_{2, i}\right], \\
\lambda_{2, i}= & \kappa_{2}+\lambda_{1, i+1}\left[\mu+\left(\beta_{1}+u_{2, i}\right) \frac{N_{i} A_{i}}{P_{i}^{2}}\right] \\
& +\lambda_{2, i+1}\left[1-\delta-\gamma_{2, i}-\left(\left(\beta_{1}+\left(1-\alpha_{1}\right) u_{1, i}\right) N_{i}+\gamma_{1}\left(N_{i}+A_{i}\right)\right) \frac{A_{i}}{P_{i}^{2}}\right] \\
& +\lambda_{3, i+1}\left[\left(\gamma_{1}\left(N_{i}+A_{i}\right)-\alpha_{1} u_{1, i} N_{i}\right) \frac{A_{i}}{P_{i}^{2}}+\gamma_{2, i}\right], \\
\lambda_{3, i}= & \kappa_{1}+\lambda_{1, i+1}\left[\mu+\epsilon_{2}-\left(\beta_{1}+u_{1, i}\right) \frac{N_{i}\left(N_{i}+S_{i}\right)}{P_{i}^{2}}\right] \\
& +\lambda_{2, i+1}\left[\epsilon_{1}+\left(\left(\beta_{1}-\left(\alpha_{1}-1\right) u_{1, i}\right) N_{i}-\gamma_{1} S_{i}\right) \frac{\left(N_{i}+S_{i}\right)}{P_{i}^{2}}\right] \\
& +\lambda_{3, i+1}\left[1-\delta-\epsilon_{1}-\epsilon_{2}+\left(\alpha_{1} u_{1, i} N_{i}+\gamma_{1} S_{i}\right) \frac{\left(N_{i}+S_{i}\right)}{P_{i}^{2}}\right],
\end{aligned}
$$

with the transversality conditions at time $T$

$$
\begin{aligned}
& \lambda_{1, T}=\kappa_{3}, \\
& \lambda_{2, T}=\kappa_{2}, \\
& \lambda_{3, T}=\kappa_{1} .
\end{aligned}
$$

Moreover, for $i=1,2,3,4 \ldots, T-1$, the optimal control $u_{1, i}^{*}$ and $u_{2, i}^{*}$ can be computed as

$$
\begin{aligned}
& u_{1, i}^{*}=\min \left\{1, \max \left\{0, \frac{N_{i} A_{i}}{P_{i} \kappa_{4}}\left(\lambda_{1, i+1}+\lambda_{2, i+1}\left(\alpha_{1}-1\right)-\alpha_{1} \lambda_{3, i+1}\right)\right\}\right\}, \\
& u_{2, i}^{*}=\min \left\{1, \max \left\{0, \frac{N_{i}}{\kappa_{5}}\left(\lambda_{1, i+1}+\lambda_{2, i+1}\left(\alpha_{2}-1\right)-\alpha_{2} \lambda_{3, i+1}\right)\right\}\right\},
\end{aligned}
$$


Proof. The Hamiltonian at time step $i$ is given by

$$
\begin{aligned}
\mathcal{H}_{i}= & \kappa_{1} A_{i}+\kappa_{2} S_{i}-\kappa_{3} N_{i}+\frac{\kappa_{4}}{2} u_{1, i}^{2}+\frac{\kappa_{5}}{2} u_{2, i}^{2} \\
& +\lambda_{1, i+1}\left[\left(1-\delta-\beta_{2, i}-u_{2, i}\right) N_{i}+\mu P_{i}-\left(\beta_{1}+u_{1, i}\right) \frac{N_{i} A_{i}}{P_{i}}+\epsilon_{2} A_{i}\right] \\
& +\lambda_{2, i+1}\left[\left(1-\delta-\gamma_{2, i}\right) S_{i}+\left(\beta_{1}+\left(1-\alpha_{1}\right) u_{1, i}\right) \frac{N_{i} A_{i}}{P_{i}}\right. \\
& \left.+\left(\beta_{2, i}+\left(1-\alpha_{2}\right) u_{2, i}\right) N_{i}-\gamma_{1} \frac{S_{i} A_{i}}{P_{i}}+\epsilon_{1} A_{i}\right] \\
& +\lambda_{3, i+1}\left[\left(1-\delta-\epsilon_{1}-\epsilon_{2}\right) A_{i}+\gamma_{1} \frac{S_{i} A_{i}}{P_{i}}+\gamma_{2, i} S_{i}+\alpha_{1} u_{1, i} \frac{N_{i} A_{i}}{P_{i}}+\alpha_{2} u_{2, i} N_{i}\right] .
\end{aligned}
$$

For $i=0,1,2, \ldots, T-1$, the adjoint equations and transversality conditions can be obtained by using Pontryagin's maximum principle in discrete time which given in [9, 11-14]

$$
\begin{aligned}
\lambda_{1, i}=\frac{\partial \mathcal{H}_{i}}{\partial N_{i}}, & \lambda_{1, T}=\kappa_{3}, \\
\lambda_{2, i}=\frac{\partial \mathcal{H}_{i}}{\partial S_{i}}, & \lambda_{2, T}=\kappa_{2}, \\
\lambda_{3, i}=\frac{\partial \mathcal{H}_{i}}{\partial A_{i}}, & \lambda_{3, T}=\kappa_{1} .
\end{aligned}
$$

For $i=0,1,2, \ldots, T-1$, the optimal controls $u_{1, i}^{*}$ and $u_{2, i}^{*}$ can be solved from the optimality condition,

$$
\begin{aligned}
\frac{\partial \mathcal{H}_{i}}{\partial u_{1, i}^{*}} & =\kappa_{4} u_{1, i}-\frac{N_{i} A_{i}}{P_{i}}\left(\lambda_{1, i+1}+\lambda_{2, i+1}\left(\alpha_{1}-1\right)-\alpha_{1} \lambda_{3, i+1}\right)=0, \\
\frac{\partial \mathcal{H}_{i}}{\partial u_{2, i}^{*}} & =\kappa_{5} u_{2, i}-N_{i}\left(\lambda_{1, i+1}+\lambda_{2, i+1}\left(\alpha_{2}-1\right)-\alpha_{2} \lambda_{3, i+1}\right)=0,
\end{aligned}
$$

we have

$$
\begin{aligned}
& u_{1, i}=\frac{N_{i} A_{i}}{P_{i} \kappa_{4}}\left(\lambda_{1, i+1}+\lambda_{2, i+1}\left(\alpha_{1}-1\right)-\alpha_{1} \lambda_{3, i+1}\right), \\
& u_{2, i}=\frac{N_{i}}{\kappa_{5}}\left(\lambda_{1, i+1}+\lambda_{2, i+1}\left(\alpha_{2}-1\right)-\alpha_{2} \lambda_{3, i+1}\right) .
\end{aligned}
$$

By the bounded in $\mathbf{U}$ of the controls, it is easy to obtain $u_{1, i}^{*}$ and $u_{2, i}^{*}$ in the form of Eqs. (4.4) and (4.5). 


\section{NUMERICAL SIMULATION}

\subsection{Algorithm}

In this section, we solve numerically the optimal control problem of the state and adjoint equations Eqs. (3.1) and (4.2). The proposed optimal control strategy is illustrated by solving the optimal system that contains six difference equations and initial and boundary conditions. So, the optimality system is given by the following.

Step 1. Providing initial and boundary conditions: $N_{0}=N_{0}, S_{0}=S_{0}, A_{0}=A_{0}, \lambda_{1, T}=$ $\kappa_{3}, \lambda_{2, T}=\kappa_{2}, \lambda_{3, T}=\kappa_{1}$ and given $u_{1,0}^{*}$ and $u_{2,0}^{*}$.

Step 2. The six optimal system in Eqs. (3.1) and (4.2) for $i=0,1,2, \ldots, T-1$ can be solve using the iterative method by using an initial guess for the control variables $u_{1,0}^{*}$ and $u_{2,0}^{*}$ in step 1 , the state variables $N, S, A$ in step 2 can be solved forward difference, but the adjoint variables $\lambda_{i}, i=1,2,3$ are solved backward difference at time steps $i=0$ and $i=T$. If the new values of the state and adjoint variables differ from the previous values, then the new values are recalculate to update $u_{1, i}$ and $u_{2, i}$ and the process is repeated until the system converges as $N_{i}^{*}=N_{i}, S_{i}^{*}=S_{i}, A_{i}^{*}=A_{i}, u_{1, i}^{*}=$ $u_{1, i}, u_{2, i}^{*}=u_{2, i}$. f or $i=0,1,2, \ldots, T-1$,

Since the optimality system is a two-point boundary value problem with separated boundary conditions at times step $i=0$ and $i=T$. The numerical solution is executed using MATLAB with the following parameter values and initial values of the state variable in Table 5.1.

\begin{tabular}{|c||l||c|}
\hline Parameters & Values used & Reference \\
\hline$\mu$ & 0.000833 & {$[2]$} \\
$\delta$ & 0.000666 & {$[2]$} \\
$\beta_{1}$ & 0.002453 & {$[2]$} \\
$\beta_{2}$ & $(0.2) * \zeta *(0.25 * 0.02)$ & Estimated \\
$\gamma_{1}$ & 0.0048 & {$[2]$} \\
$\gamma_{2}$ & $(0.2) * \zeta *(0.25 * 0.02)+k$ & Estimated \\
$\epsilon_{1}$ & 0.0020 & {$[2]$} \\
$\epsilon_{2}$ & 0.000634 & {$[2]$} \\
$\alpha_{1}$ & 0.5 & Estimated \\
$\alpha_{2}$ & 0.5 & Estimated \\
\hline
\end{tabular}

Table 5.1: The value of parameters for Discrete-time model. 


\subsection{First strategy}

In the first strategy, influencer marketing campaign $\left(u_{1}\right)$ is used. Meanwhile marketting capaign is not used $\left(u_{2}=0\right)$. The profile of the ooptimal control $u_{1}$ is plotted in Figure 5.1. The influencer marketing campaign should be done intensively for almost 10 months and then decreasing in months 10.

Furthermore, the dynamics of rational buyers are given in Figure 5.2, the dynamics of excessive buyers are given in Figure 5.3, and the dynamics of addictive buyers are given in Figure 5.4. Figure 5.4 shows that addictive buyers for influencer marketing campaign controls provide an increment in addictive buyers compared to having no controls.

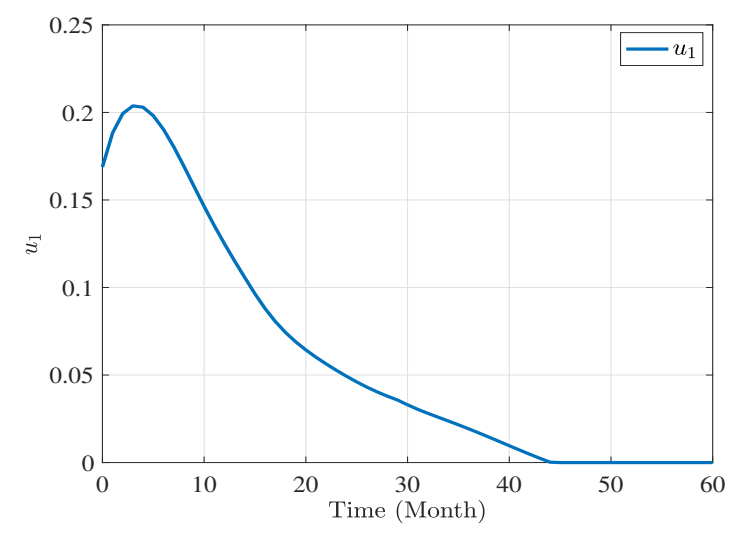

Figure 5.1: Profile of optimal control $u_{1}$.

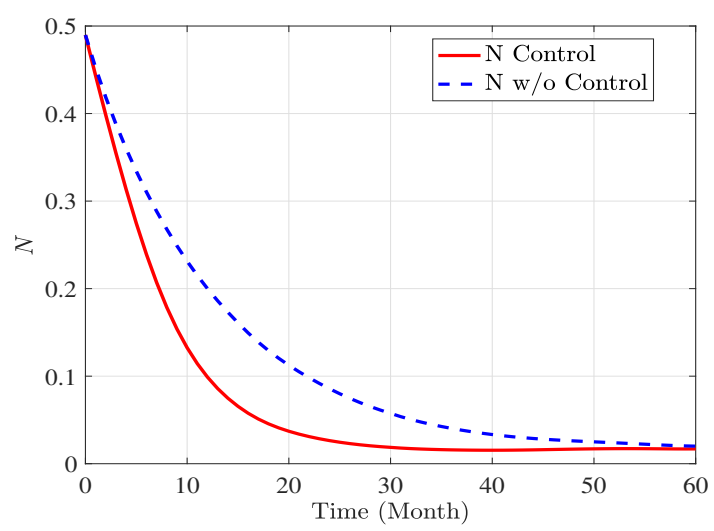

Figure 5.2: The simulations concerning rational buyers using control $u_{1}$. 


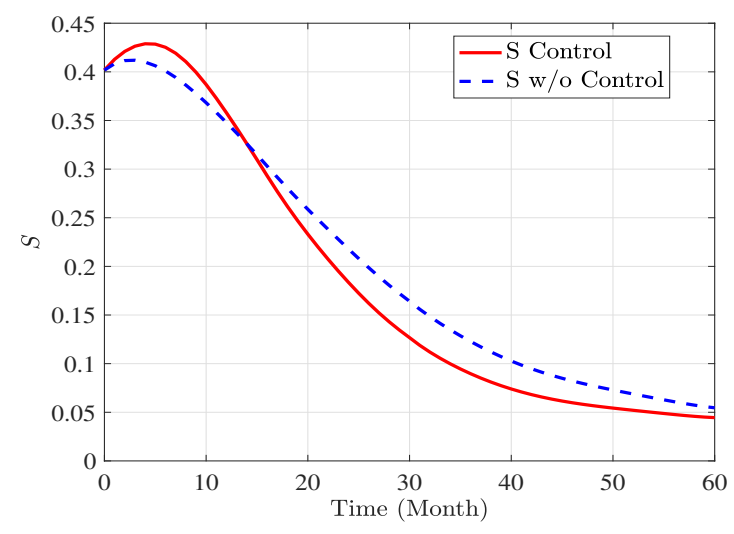

Figure 5.3: The simulations concerning excessive buyers using control $u_{1}$.

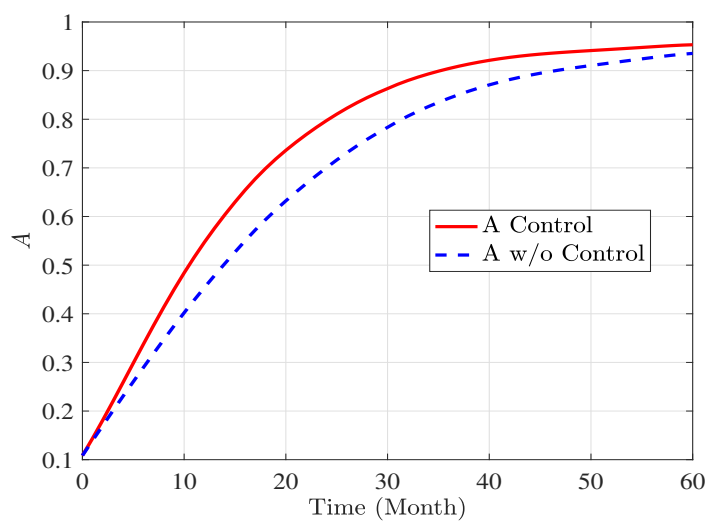

Figure 5.4: The simulations concerning addictive buyers using control $u_{1}$.

\subsection{Second strategy}

In the second strategy, we implemented of optimal control for voucher marketing campaign $\left(u_{2}\right)$ for simulation. The profile of the optimal control $\left(u_{2}\right)$ is given in Figure 5.5. The voucher marketing campaign should be done intensively then decreasing in the next month.

Furthermore, the dynamics of rational buyers are given in Figure 5.6, the dynamics of excessive buyers are given in Figure 5.7, and the dynamics of addictive buyers are given in Figure 5.8. Figure 5.8 shows that addictive buyers for voucher marketing campaign controls provide an increment in addictive buyers compared to having no controls. 


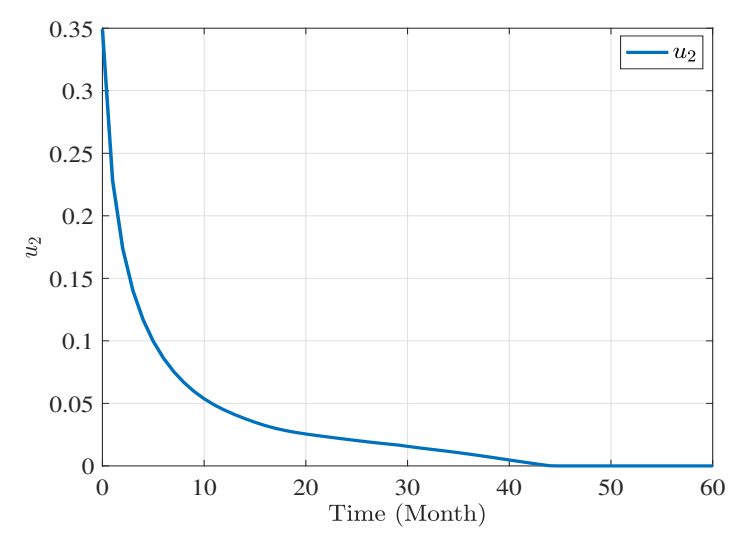

Figure 5.5: Profile of optimal control $u_{2}$.

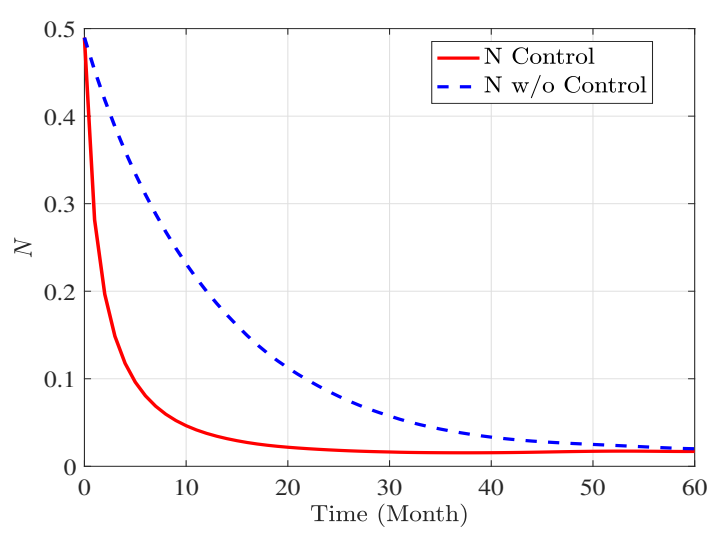

Figure 5.6: The simulations concerning rational buyers using control $u_{2}$.

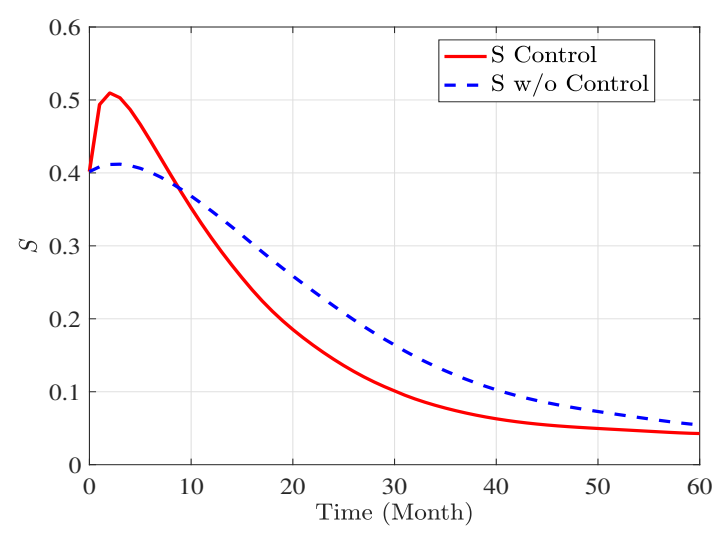

Figure 5.7: The simulations concerning excessive buyers using control $u_{2}$.

\subsection{Third strategy}

In the third strategy, we implement the combination of the optimal control for the influencer marketing campaign $\left(u_{1}\right)$ and voucher marketing campaign $\left(u_{2}\right)$ for 


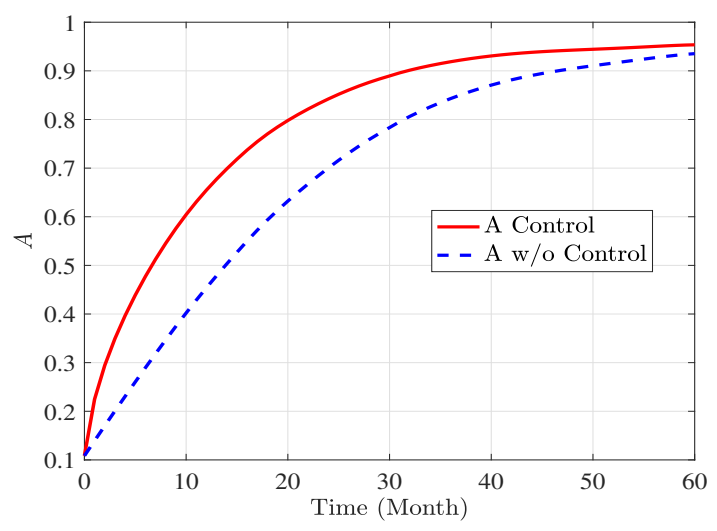

Figure 5.8: The simulations concerning addictive buyers using control $u_{2}$.

simulations. The profile of optimal controls $\left(u_{1}\right)$ and $\left(u_{2}\right)$ is plotted in Figure 5.9. The influencer marketing campaign should be done intensively in the beginning then decreasing in the next month. Meanwhile, the voucher marketing campaign should be done intensively likewise.

Figures 5.10 and 5.12 show the dynamics of rational buyer and addictive buyer in the populations, respectively, using the optimal controls $\left(u_{1}\right)$ and $\left(u_{2}\right)$. In utilizing this strategy, the rational buyer in the populations decreases more than it previous strategy and addictive buyer in the populations increase when using this strategy.

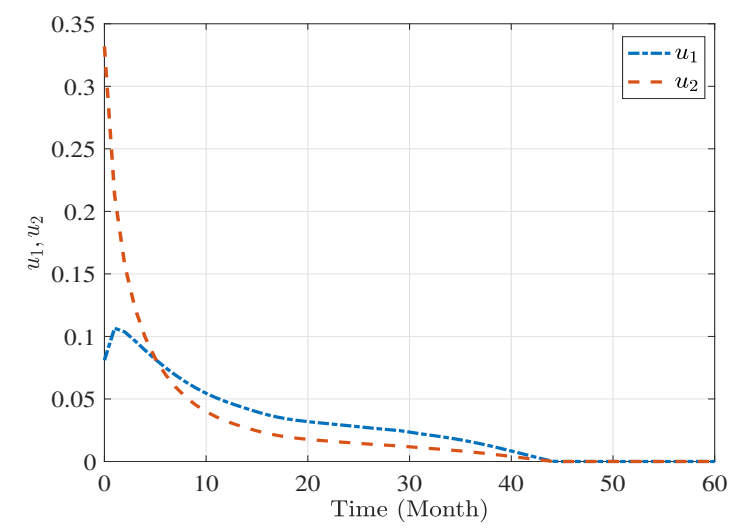

Figure 5.9: Profile of optimal controls $u_{1}$ and $u_{2}$.

\section{CONCLUSION}

In this paper, we will show you the best controlled mathematical model related to addictive buying, with two controls. The first is control using an influencer marketing campaign to promote marketing advertising and the second is to use a voucher marketing campaign distribution in the market to entice the population to become more 


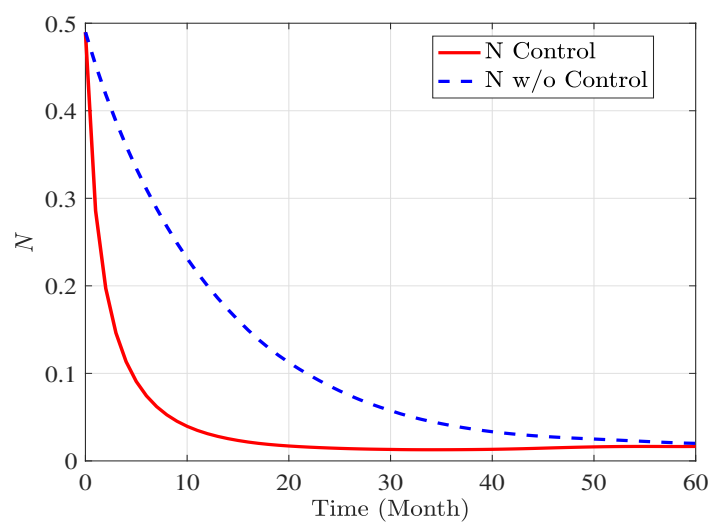

Figure 5.10: The simulations concerning rational buyers using controls $u_{1}$ and $u_{2}$.

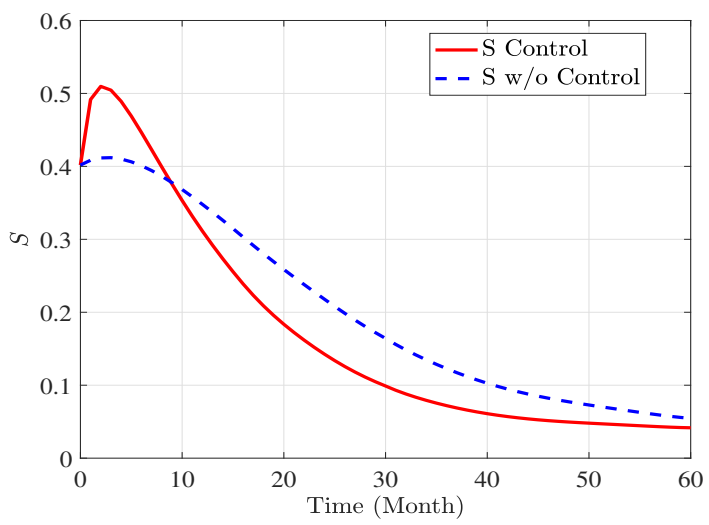

Figure 5.11: The simulations concerning excessive buyers using controls $u_{1}$ and $u_{2}$.

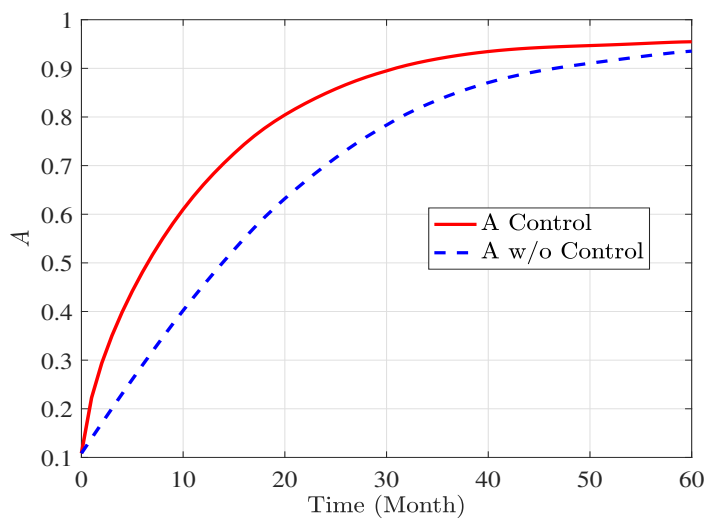

Figure 5.12: The simulations concerning addictive buyers using controls $u_{1}$ and $u_{2}$.

addictive buying. From the results of this model, it is found that, if using only control, should use voucher marketing campaign distribution, which will make the population more addictive. Rather than using the use of influencer marketing campaigns to help 
promote advertisements in marketing. But if it is best to use both types of controls at the same time, there will be many variations in the advertisement promotions. Using a cost-effective program is done through the method of sweeping forward, backward in the equation derived from necessary conditions. Increasing the efficiency of addictive buyers while reducing the cost of using both controls, on the other hand, effectively reducing both rational buyers and excessive buyers.

\section{Acknowledgment}

This research is partially supported by the Center of Excellence in Mathematics, the Commission on Higher Education, Thailand and the Department of Mathematics, King Mongkut's University of Technology North Bangkok, Thailand.

\section{REFERENCES}

[1] C. Tyagi and A. Kumar, Consumer behaviour. Atlantic Publishers \& Dist, 2004.

[2] I. García, L. Jódar, P. Merello, and F.-J. Santonja, “A discrete mathematical model for addictive buying: predicting the affected population evolution," Mathematical and Computer Modeling, vol. 54, no. 7-8, pp. 1634- 1637, 2011.

[3] Thiti P, Sanoe K, Walailuck C. "A mathematical model of optimal control for addictive buying: predicting the population behavior", 9(3), COMPUSOFT, An International Journal of Advanced Computer Technology. PP. 3611-3616.

[4] C. M. Silva, S. Rosa, H. Alves, and P. G. Carvalho, "A mathematical model for the customer dynamics based on marketing policy," Applied Mathematics and Computation, vol. 273, pp. 42-53, 2016.

[5] P. Upadhyay, S. Upadhyay, and K. Shukla, "A mathematical model of consumers' buying behaviour based on multiresolution analysis," Procedia computer science, vol. 122, pp. 564-571, 2017.

[6] K. Chaudhary and P. C. Jha, "Optimal control promotional policy for a new product incorporating repeat purchase in segmented market: A control theoretic approach," in Industrial Engineering, IntechOpen, 2018.

[7] J. Foster, C. Deck, and A. Farmer, "Behavioral demand effects when buyers anticipate inventory shortages," European Journal of operational research, vol. 276, no. 1, pp. 217-234, 2019.

[8] “Thailandconsumerconfidence $|2019|$ data|chart|calendar|forecast.” https://trading economics.com/thailand/consumer-confidence. 
[9] W. Ding, R. Hendon, B. Cathey, E. Lancaster, and R. Germick, "Discrete time optimal control applied to pest control problems," Involve, a Journal of Mathematics, vol. 7, no. 4, pp. 479-489, 2014.

[10] S. Rosa, P. Rebelo, C. M. Silva, H. Alves, and P. G. Carvalho, "Optimal control of the customer dynamics based on marketing policy," Applied Mathematics and Computation, vol. 330, pp. 42-55, 2018.

[11] 10. D. Zhang and B. Shi, "Oscillation and global asymptotic stability in a discrete epidemic model," Journal of Mathematical Analysis and Applications, vol. 278, no. 1, pp. 194-202, 2003.

[12] V. Guibout and A. Bloch, "A discrete maximum principle for solving optimal control problems," in 2004 43rd IEEE Conference on Decision and Control (CDC)(IEEE Cat. No. 04CH37601), vol. 2, pp. 1806-1811, IEEE, 2004.

[13] C. Hwang and L. Fan, "A discrete version of pontryagin's maximum principle," Operations Research, vol. 15, no. 1, pp. 139-146, 1967.

[14] M. Alipour, M. A. Vali, "Appling Homotopy Analysis Method to Solve Optimal Control Problems Governed by Volterra Integral Equations", Journal of Computer Science \& Computational Mathematics, vol. 5, no. 3, pp. 41-47, 2015. 